\title{
DRUG UTILIZATION PATTERN OF PSYCHOTROPIC DRUGS IN PSYCHIATRIC OUTPATIENT DEPARTMENT IN A TERTIARY CARE TEACHING HOSPITAL
}

\author{
LETTY SABU ${ }^{1}$, MARIYA YACOB ${ }^{1}$, MAMATHA ${ }^{1 *}$, HEMENDRA SINGH ${ }^{2}$
}

${ }^{1}$ Department of Pharmacy Practice, Faculty of Pharmacy, M. S. Ramaiah University of Applied Sciences, Bengaluru, Karnataka, India. ${ }^{2}$ Department of Psychiatry, M. S. Ramaiah Medical College and Hospitals, Bengaluru, Karnataka, India.

ABSTRACT

Objective: The growing concern over the burden of psychiatric illnesses in health statistics elicit the importance of rational prescribing of psychotropic drugs. Due to the minimal number of studies conducted in this area, the present study was carried out to assess the morbidity pattern and prescribing trend of drugs used in psychiatric outpatients.

Methods: This prospective observational study was conducted for a period of 6-month in a psychiatric outpatient department. The medical records of 202 patients were reviewed for analyzing the prescription pattern using the World Health Organization drug indicators, wherein patients were categorized according to the International Classification of Diseases-10 criteria.

Results: In the present study, 129 (63.9\%) of the 202 patients were males. The majority of the patients (36.63\%) were in the age group of 25 -35 years. Substance-related disorders (28.71\%) were the most common psychiatric disorders followed by schizophrenia and other psychotic disorders $(22.77 \%)$. Anxiolytics and hypnotics (31.99\%) were the most commonly prescribed psychotropic drug class followed by antipsychotics (20.2\%) and antidepressants (18.18\%) with clonazepam (47.89\%), risperidone (42.5\%), and escitalopram (57.4\%) being the most common drugs, respectively. Polypharmacy was found in $93.56 \%$ of the prescriptions with an average of 3.396 drugs/prescription. Generic drug usage (39.94\%) was found to be low when compared to the brand drug usage $(60.05 \%)$.

Conclusion: Benzodiazepines were the most commonly used psychotropic drugs. Polypharmacy, although practiced as a treatment strategy to manage psychiatric illnesses, predisposes patients to adverse events and adherence issues.

Keywords: Drug utilization pattern, Psychotropic drugs, Polypharmacy, Psychiatric outpatients.

(c) 2017 The Authors. Published by Innovare Academic Sciences Pvt Ltd. This is an open access article under the CC BY license (http://creativecommons. org/licenses/by/4. 0/) DOI: http://dx.doi.org/10.22159/ajpcr.2017.v10i1.15112

\section{INTRODUCTION}

Psychiatric disorders are gaining importance in today's world which is governed by globalization and competition. The National Institute of Family Welfare in India states that $2-3 \%$ of the population is suffering from serious debilitating mental disorders or epilepsy and about $10.4-53 \%$ visit general medicine outpatient department (OPD) with mental conditions. The majority of the psychiatric population needs long-term or even life-long therapy with psychotropics. However, these drugs often affect emotion and cognition wherein significant clinical, legal, and psychological issues are being observed $[1,2]$

In a developing country like India, where almost all psychotropic medications are available, very few studies have been conducted to evaluate the psychotropic drug utilization pattern [3]. The World Health Organization (WHO) has defined drug utilization study as a study of marketing, distribution, prescription, and use of drugs in society, with special emphasis on the resulting medical, social, and economic implications [4]. Drug utilization studies provide insight into the efficacy of drugs and show variations in prescribing trends and pattern of polypharmacy $[5,6]$. The most common reasons behind multiple drug use or polypharmacy are to treat two pathophysiologically yet distinct but comorbid illness, augmentation of the efficacy of primary treatment, reduction or management of adverse effects of primary treatment [6]. However, with increased treatment regimen complexity, concerns regarding possibility of cumulative toxicity, undesirable side effects as well as adherence issues emerge with polypharmacy [7]. Medication nonadherence, in turn, may increase the risk of relapse and rehospitalization which can have a major impact on the course of illness and treatment outcomes [8].

Even though the incidence of polypharmacy and adverse drug reactions have been substantially reduced with the usage of the newer generation of psychotropics, extensive studies on their prescribing and usage need to be carried out $[9,10]$. Hence, the present study was conducted to analyze the drug utilization pattern of psychotropics in the psychiatric OPD at a tertiary care teaching hospital.

\section{METHODS}

This hospital-based prospective observational study was carried out in outpatients of the Psychiatry Department of M. S. Ramaiah Medical Teaching Hospital (MSRMTH), Bengaluru, Karnataka, for a period of 6-month from January 2015 to June 2015. The complete project was done in accordance with the permission granted by MSRMTHs Ethics Committee. Patients diagnosed with a psychiatric disorder, regardless of sex, and comorbidities were enrolled as per inclusion and exclusion criteria. Patient's case notes, medication charts, lab reports, previous outpatient and inpatient records, and other relevant documents were reviewed, and details were recorded in a predesigned structured pro forma to analyze the drug utilization pattern. The Statistical Package for the Social Science (SPSS) Version 20.0 was used to analyze data. The WHO drug use indicators were used for analyzing the prescriptions. 


\section{Inclusion criteria}

Patients of either gender attending the OPD of psychiatric setting and patients treated with one or more psychopharmacological agents in psychiatric OPD were selected.

\section{Exclusion criteria}

Patients treated on an inpatient basis; patients treated with nonpsychopharmacological therapy, patients who were agitated and uncooperative were excluded from the study.

\section{RESULTS}

A total of 202 patients satisfied inclusion criteria and a male preponderance $(63.86 \%)$ was observed in the study population. The majority of patients belonged to the age group of $25-35$ years (36.63\%). Morbidity pattern showed increased frequency with substance-related disorders $(28.71 \%)$ followed by schizophrenia and other psychotic disorders $(22.77 \%)$ and anxiety disorders (18.81\%) (Fig. 1). Benzodiazepines (BZDs) (28.96\%), antidepressants $(18.18 \%)$, and atypical antipsychotics $(18.18 \%)$ were the most common categories of drugs prescribed.

The most frequently prescribed BZDs have been clonazepam (47.89\%), diazepam (21.05\%), and lorazepam (18.95\%). Among the antidepressants, escitalopram $(57.4 \%)$ and sertraline $(12.96 \%)$ were the most commonly prescribed drugs. Risperidone accounted for about $42.5 \%$ of the prescribed antipsychotics followed by olanzapine $(23.33 \%)$ and quetiapine (15\%). Among the anticonvulsants, topiramate (36.58\%) and valproic acid (36.58\%) were frequently prescribed. Vitamin and mineral supplements $(63.7 \%)$ followed by trihexyphenidyl (20.74\%) were the most commonly prescribed drugs among other psychotropic drugs (Table 1).

A total of 202 prescriptions containing 686 drugs were analyzed. Polypharmacy was found in $93.56 \%$ of the prescriptions with an average of 3.396 drugs/prescription. Polypharmacy was found to be more among the age group 25-35 years (34.39\%). Drugs prescribed in their generic name constituted $39.94 \%$ while the prescriptions with injectable drugs accounted for $2.04 \%$ and $0.01 \%$ of the prescription contained psychotropic fixed dose combination (FDC). $27.98 \%$ of the psychotropic drugs were prescribed from WHO's 18 ${ }^{\text {th }}$ List of Essential Medicines (Table 2)

\section{DISCUSSION}

Psychotropics remain a mainstay in the treatment of psychiatric illness. Drug utilization studies of psychotropic drugs reflect the present trends in therapeutic decision making. In this study, a total of 202 prescriptions were analyzed, of which, 129 (63.86\%) were males and 73 (36.13\%) were females which is in concordance with the study conducted by Kumar et al. [11]. The male preponderance in the present study can be attributed to the morbidity pattern observed with substance-related disorders (alcohol dependence syndrome and nicotine dependence syndrome) as the most common psychiatric disorder

According to present findings, the majority of the patients belonged to 25-35 years age group which was found similar to the study conducted by Sarumathy et al., wherein more than $66 \%$ of patients belonged to 21-40 years of age group [12]. Morbidity pattern among psychiatric disorders showed substance-related disorders (28.71\%) as the most common psychiatric disorder followed by schizophrenia and other psychotic disorders $(22.77 \%)$. This is in contrary to the multicentric study conducted by the Indian Psychiatric Society where affective disorders followed by neurotic, stress-related, and somatoform disorders were the most common diagnostic categories [13]. Higher prevalence of substance use disorders in the present study may be probably due to increase help-seeking behavior for the same in the study population.

The drug utilization pattern in the study population showed BZDs $(28.96 \%)$ as the most commonly prescribed psychotropic drug class

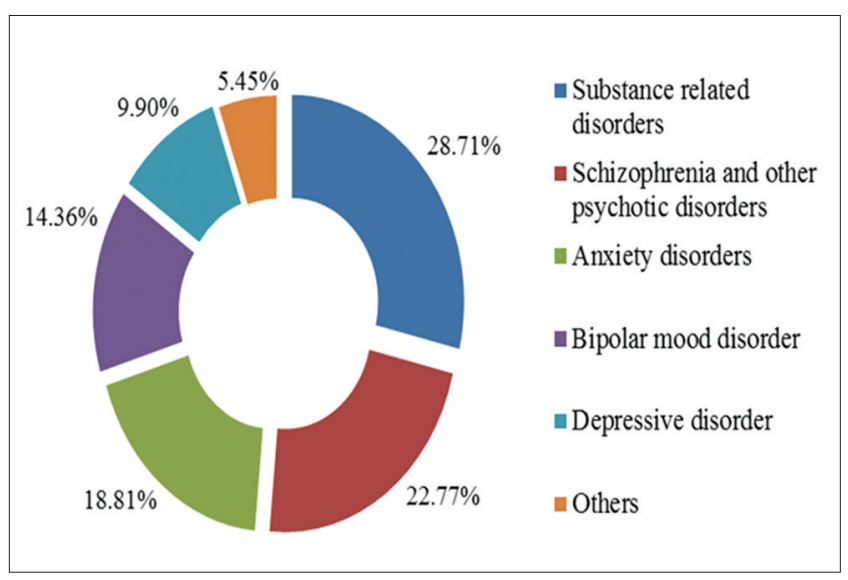

Fig. 1: It depicts distribution of psychiatric disorders

Table 1: Utilization of psychotropics in psychiatric OPD

\begin{tabular}{ll}
\hline Drug class & Number of drugs (\%) \\
\hline Antidepressants & $108(18.18)$ \\
Atypical antipsychotics & $108(18.18)$ \\
Typical antipsychotics & $12(2.02)$ \\
Benzodiazepines & $172(28.96)$ \\
Nonbenzodiazepine hypnotics & $18(3.03)$ \\
Anticonvulsants & $41(6.90)$ \\
Anticholinesterases & $6(1.01)$ \\
Antimuscarinics & $26(4.38)$ \\
Mood stabilizers & $14(2.356)$ \\
Vitamin and mineral supplements & $89(14.99)$ \\
Total & $594(100)$ \\
\hline
\end{tabular}

OPD: Outpatient department

Table 2: Drug use pattern of psychotropics in psychiatric OPD

\begin{tabular}{ll}
\hline Drug use indicators & Result \\
\hline Total number of prescriptions analyzed & 202 \\
Total number of drugs prescribed & 686 \\
$\begin{array}{l}\text { Average number of the drugs per prescription } \\
\text { Average number of the psychotropic per drugs per } \\
\text { prescription }\end{array}$ & 3.396 \\
$\begin{array}{l}\text { Percentage of the psychotropic drugs prescribed by } \\
\text { generic name (\%) }\end{array}$ & 2.56 \\
$\begin{array}{l}\text { Percentage of injectable drugs prescribed (\%) } \\
\text { Percentage of the prescriptions containing psychotropic }\end{array}$ & 2.94 \\
$\begin{array}{l}\text { FDC (\%) } \\
\text { Percentage of psychotropic drugs prescribed from }\end{array}$ & 0.01 \\
essential drug list (\%) & 27.98 \\
\hline
\end{tabular}

OPD: Outpatient department, FDC: Fixed dose combination

followed by antipsychotics (18.18\%) and antidepressants (18.18\%). In our study, more than half of the psychotic patients received adjunctive BZD, clonazepam $(47.89 \%)$ being the most common BZD followed by diazepam $(21.05 \%)$ and lorazepam (18.95\%). Similar findings were found in a study carried out by Rode et al., which showed the prescribing frequency of anxiolytics, antidepressants, antipsychotics, anticholinergics, and antimania drugs as $30.04 \%, 25.46 \%, 25.37 \%$, $11.54 \%$, and $7.6 \%$, respectively [14].

Prescribing pattern of antipsychotics in our study showed increased usage of atypical antipsychotics (18.8\%) compared to typical antipsychotics $(2.02 \%)$. Risperidone $(42.5 \%)$ was the most commonly prescribed antipsychotic followed by olanzapine (23.33\%). Prescriptions consisting of typical antipsychotics were found to be less, wherein chlorpromazine and haloperidol being the only drugs prescribed ( $5 \%$ each). This is similar to the results from the preliminary 
survey on antipsychotic prescribing pattern conducted among Indian Psychiatrists [15]. The most commonly prescribed antidepressant medication according to our study was escitalopram (57.40\%) followed by sertraline $(12.96 \%)$ which was similar to the findings from a multicentric study conducted by the Indian Psychiatry Society [13]. However, among the mood stabilizers, lithium and sodium valproate were commonly prescribed, followed by carbamazepine which is in contrary to study conducted by the Indian Psychiatry Society and in concordance with the study conducted by Trivedi et al. $[13,16]$. The WHO guidelines state that the number of antipsychotic drugs per prescription should be within 1.6-1.8/encounter, our study showed 3.39 , which is considerably more. In our study, around $60 \%$ of the prescriptions consisted of $1-3$ drugs and $40 \%$ of the prescriptions contained four or more drugs. These findings suggested a rise in the incidence of polypharmacy with high prevalence among 25-35 years age group which is supported by a study conducted by Deshmukh and Ismail [5]. Polypharmacy can lead to poor compliance, drug interactions, adverse drug reactions, under-use of effective treatments and medication errors [17]. The reason for polypharmacy in our study may be attributed to the complexity of presentation in psychiatric illness and the presence of comorbidities. Generic drug usage reduces the overall cost of therapy, but our study revealed a low percentage of generic drug usage (39.94\%) compared to brand drug usage (60.06\%). The prescriptions containing psychotropic FDCs were very minimal $0.01 \%$ which may be due to unavailability of expected FDC in the market. Concerns about the adverse effects and cost-effectiveness of parenteral routes of drug administration are probably the reason for the low utilization of "depot injection" formulation in the study (2.04\%). The percentage of drugs prescribed from the WHO Essential Drug List was found to be 27.98, and the primary purpose of WHO Essential Drug List is to promote rational use of medicines considering the three important aspects, i.e., cost, safety, and efficacy. Our study suggests a strong need for creating awareness in the prescribers regarding the usage of generic drugs as they are cost-effective compared to brand drugs.

\section{CONCLUSION}

Continuous monitoring of the prescription gives an insight of present trends in prescribing patterns of psychotropic drugs in hospital settings. The drug use pattern from the study largely conforms to the standard recommendations of WHO. The commonly used psychotropic drug in the present study was BZDs. The incidence of polypharmacy should be minimized in psychiatric outpatients as it might result in poor compliance, increased drug interactions, and side effects. The generic drug usage is a rational prescribing indicator, and the issue of brand drug use needs to be addressed. Our study suggests a strong need for creating awareness in the prescribers regarding the usage of generic drugs as they are cost-effective compared to brand drugs. Our study was carried out for a short duration and study subjects were largely confined to OPD. Thus, the present study provides a baseline to conduct drug utilization studies periodically for a longer duration in a larger sample size to influence the overall improvement of patients on psychotropic drugs in different mental health care settings.

\section{ACKNOWLEDGMENT}

The authors are grateful to the Department of Psychiatry, MSRMTH, for their constant support and guidance.

\section{REFERENCES}

1. Jayanthi CR, Divyashree M, Sushma M. Adverse drug reactions in psychiatry outpatients: Clinical spectrum, causality and avoidability. J Chem Pharm Res 2013;5(8):128-35.

2. Prueksaritanond S, Tubtimtes S, Pumkompol T, Sukying C. Psychotropic drug prescribing in the family medicine out-patient clinic, Ramathibodi Hospital. J Med Assoc Thai 2009;92(2):266-72.

3. Shamkuwar CA, Chakravorty AD, Shrivastava MP, Deshmukh R. Pattern of prescription and drug use in psychiatry outpatient department of private practitioners of central India. Int J Basic Clin Pharmacol 2013;2(6):777-82.

4. WHO International Working Group for Drug Statistics Methodology. Introduction to Drug Utilization Research. Geneva: WHO Collaborating Centre for Drug Utilization Research and Clinical Pharmacology; 2003. Available from: http://www.whocc.no/filearchive/publications/drug utilization research.pdf. [Last cited on 2012 Nov 01].

5. Deshmukh SA, Ismail T. Evaluation of psychotropic drugs use pattern among outpatients attending psychiatry department at government medical college and hospital, Nagpur: A cross sectional study. Int J Pharm Bio Sci 2012;3(3):428-36.

6. Sarkar P, Chakraborty K, Misra A, Shukla R, Swain SP. Pattern of psychotropic prescription in a tertiary care center: A critical analysis. Indian J Pharmacol 2013;45(3):270-3.

7. Sanjay K, Gurvinder K, Nilesh S, Amresh S. Polypharmacy in psychiatry: A review. Mens Sana Monogr 2013; 1(1):82-9.

8. Nirojini SP, Bollu M, Nadendla RR. Prevalence of medication non-adherence among the psychiatric patients - results from a survey conducted in a tertiary care hospital. Int J Pharm Pharm Sci 2014;6(4):461-3.

9. Paul PK, Konwar M, Das S. To study the prescribing pattern of antipsychotic drugs in a tertiary care hospital of Assam. Int J Pharm Pharm Sci 2014;6(4):435-7.

10. Chattar KB, Karve AV, Subramanyam A, Tondare SB. Prescription pattern analysis of antidepressants in psychiatric outpatient department of tertiary care hospital in India. Asian J Pharm Clin Res 2016;9(4):77-9.

11. Kumar SP, Yasmeen N, Raju YS, Gurunath S, Duvvala P. Psychiatric disorders: Prescribing patterns, cost of antipsychotics and prevalence trend from past to present. Am J Phytomed ClinTher 2013;1(7):554-61.

12. Sarumathy S, Menaka K, George PS, Ravichandiran V. A study on drug use pattern and adverse drug reactions of anti-psychiatric medications in a psychiatry specialized hospital. Int J Pharm Pharm Sci 2014;6(6):332-4.

13. Grover S, Avasthi A, Sinha V, Lakdawala B, Bathla M, Sethi S, et al. Indian psychiatric society multicentric study: Prescription patterns of psychotropics in India. Indian J Psychiatry 2014;56(3):253-64.

14. Rode SB, Ajagallay RK, Salankar HV, Sinha U. A study on drug prescribing pattern in psychiatry out-patient department from a tertiary care teaching hospital. Int J Basic Clin Pharmacol 2014;3(3):517-22.

15. Grover S, Avasthi A. Anti-psychotic prescription pattern: A preliminary survey of Psychiatrists in India. Indian J Psychiatry 2010;52(3):257-9.

16. Trivedi JK, Sareen H, Yadav VS, Rai SB. Prescription pattern of mood stabilizers for bipolar disorder at a tertiary health care centre in north India. Indian J Psychiatry 2013;55(2):131-4.

17. Thakkar KB, Jain MM, Billa G, Joshi A, Khobragade AA. A drug utilization study of psychotropic drugs prescribed in the psychiatry outpatient department of a tertiary care hospital. J Clin Diagn Res 2013;7:2759-64. 